\title{
A Note on Generalization of the Simplest Time-Dependent Discrete Markov Process: Linear Growth Process With Immigration-Emigration
}

\author{
Bijoy Kumar Pradhan ${ }^{1, *}$, Priyaranjan Dash ${ }^{1}$, Upasana \\ ${ }^{1}$ Department of Statistics, Utkal University, Bhubaneswar, Odisha, 751004, India
}

\begin{abstract}
ARTICLE INFO
Article History

Received 24 Jan 2019

Accepted 27 Apr 2020

Lineargrowth process with immigration and emigration is the general model in the study of population in biological and ecological systems, and their transient analysis is the most important factor in the understanding of the structural behavior of such systems. The probability-generating function $\pi(z, t)$ of the probability distribution $\left\{p_{n}(t)\right\}$ of the random variable $N(t)$ in many queuing situations concerning Birth, Death, Immigration, and Emigration were studied and we find the generalization of the transient solutions of the queuing systems and also studied its particular cases.
\end{abstract}

Keywords and Phrases

Birth

Death

Immigration and Emigration

Process

Linear growth process

AMS Subject Classification: 62M99, 60J80, 60J27, 60G07.

(c) 2021 The Authors. Published by Atlantis Press B.V.

This is an open access article distributed under the CC BY-NC 4.0 license (http://creativecommons.org/licenses/by-nc/4.0/).

\section{INTRODUCTION}

Transient queuing systems became the focus of the researchers since early 1940s working in the area of queuing theory and also modeling different biological populations, disease modeling specifically in the area of epidemiology but subsequently it became more popular in several areas like modeling the number of animals belonging to a particular forest, the fish population in a lake, spreading of bacteria and viruses in a specific area, logistic services like modeling operation efficiency in railway container terminal, analyzing buffer arrivals in modeling telecommunication systems, signal processing, and many more. The present paper generalizes a time-dependent Markovian process in presence of births, deaths, immigration, and emigration. The characterizing principle of such type of processes can be highlighted on the basis of the generating function of probabilities of the number of instances/occurrences. So, we focus our attention on the generating function of the process.

The generating function

$$
\pi(z, t)=\sum_{n=0}^{\infty} p_{n}(t) z^{n}
$$

for many transient queuing situations satisfy a partial differential difference equation of the form

$$
Q(z) \frac{\partial \pi(z, t)}{\partial z}+R(z) \frac{\partial \pi(z, t)}{\partial t}=1,
$$

with $p_{n}(t)=\operatorname{Pr}\{N(t)=n\}$, where $\left\{p_{n}(t)\right\}$ represents probability distribution of the random variable $N(t)$ for every value of $t$ [?].

In order to solve this partial differential difference equation subject to some appropriate boundary conditions, we are required to form the subsidiary equations given by

$$
\frac{d \pi(z, t)}{1}=\frac{d z}{Q(z)}=\frac{d t}{R(z)} .
$$

\footnotetext{
${ }^{*}$ Corresponding author. Email: prdashjsp@gmail.com
} 
Taking into considerations of the first and second parts of (1.3) and then from second and third parts of (1.3), we find two independent solutions of the subsidiary equations writing them in the form

$$
u(z, t, \pi)=\text { a constant }, \quad v(z, t)=\text { a constant. }
$$

From (1.4), we find the general solution of (1.2), i.e., the probability-generating function $\pi(z, t)$ of the probability distribution $\left\{p_{n}(t)\right\}$. We may verify that this solution satisfies the partial differential difference equation given by (1.2).

Also, one may verify that the mean and variance of the probability distribution $\left\{p_{n}(t)\right\}$ found by considering the expression for $p_{n}^{\prime}(t)$ are same as the mean and variance of the probability distribution by considering the probability-generating function $\pi(z, t)$ on the above procedure.

The present paper deals with linear growth process with immigration and emigration, where the probability-generating function is derived and its mean and variance are calculated directly by considering the expression for $p_{n}^{\prime}(t)$ and also from the the probability-generating function $\pi(z, t)$ for the probability distribution $\left\{p_{n}(t)\right\}$. We found that both procedures give the same result. From the probability-generating function $\pi(z, t)$ of the probability distribution $\left\{p_{n}(t)\right\}$ of this process, we can derive the probability-generating functions of Linear Birth Process, Linear Death Process, Birth Immigration Process, Birth Emigration Process, Death Immigration Process, Death Emigration Process, Immigration-Emigration Process, Linear Growth Process with Immigration, Linear Growth Process with Emigration, Birth process with Immigration-Emigration, Death process with Immigration-Emigration as the particular cases and also we can find the mean and variance of all these transient queuing processes from the mean and variance of linear growth process with immigration and emigration as particular cases.

Getz [1] has also found the probability-generating function by considering the wrong procedure, i.e., by considering the first and third parts of (1.3) and the second and third parts of (1.3) in his paper. The probability-generating function found by him does not satisfy the partial differential equation of this process. He found the mean and variance by considering the expressions for $p_{n}^{\prime}(t)$ of the probability distribution $\left\{p_{n}(t)\right\}$ of this process under "Direct derivation of Mean and Variance." He found the correct mean but incorrect variance. We would like to mention here that the probability-generating function given by Getz [1] is not correct.

Zheng et al. [2] have also tried to find the probability-generating function of this process. But they could not able to find the correct differential difference equation of this process because they considered additional term $\theta\left(1-\frac{1}{2}\right) P(t, 0)$ in the differential difference equation of this process (here, $\theta$ stands for Emigration rate). In this regard, we would like to mention here that the term $\theta P(t, 0)$ does not exist in the process because when the population size reduces to zero at time $t$, there will be no emigration and hence we set $\theta P(t, 0)=0$.

\section{ASSUMPTIONS}

a. If $n$ individuals are present at the instant from which the interval commences, the probability of one birth will occur in any short interval of length $h$ is $n \lambda h+o(h)$ and the probability of more than one birth occurring in that small interval is $o(h)$. Birth occurring in $(t, t+h)$ are independent of time since the last occurrence.

b. If $n$ individuals are present at the instant from which the interval commences, the probability of one death will occur in any short interval of length $h$ is $n \mu h+o(h)$ and the probability of more than one death occurring in that small interval is $o(h)$. Death occurring in $(t, t+h)$ are independent of time since the last occurrence.

c. During the small interval of time $(t, t+h)$, the probability that a new member being added to the population by immigration is $v h+o(h)$ and the probability that more than one individual is added to the population in that small interval of length $h$ is $o(h)$. Immigration occurring in $(t, t+h)$ are independent of time since the last occurrence.

d. During the small interval of time $(t, t+h)$, the probability that a member being left by emigration from the population is $\xi h+o(h)$ and the probability that more than one emigration occurring in that small interval of length $h$ is $o(h)$. Emigration occurring in $(t, t+h)$ are independent of time since the last occurrence.

e. For the same population, there is no interaction among the birth, death, immigration, and emigration in small interval $(t, t+h)$ of time.

\section{DERIVATION OF PROBABILITY-GENERATING FUNCTION}

Let $p_{n}(t)$ be the probability that the process starts with $n$ individuals at instant $t$, i.e.,

$$
p_{n}(t)=\operatorname{Pr}[N(t)=n]
$$

To calculate $p_{n}(t+h)$ at the next time point $(t+h)$, the system can be instate $E_{n}$ only if one of the following conditions are satisfied:

a. At time $t$, the population consist of $n$ individuals and no birth, no death, no immigration, no emigration occur during the time interval $(t, t+h)$.

b. At time $t$, the population consist of $(n-1)$ individuals and a birth occurs during the time interval $(t, t+h)$ but no death, no immigration, no emigration occur during that interval of time. 
c. At time $t$, the population consist of $(n-1)$ individuals and a new member is added to the population by immigration during the time interval $(t, t+h)$ but no birth, no death, no emigration occur during that interval of time.

d. At time $t$, the population consist of $(n+1)$ individuals and a death occurs during the next time interval $(t, t+h)$ but no birth, no immigration, no emigration occur during that interval of time.

e. At time $t$, the population consist of $(n+1)$ individuals and an individual left the process during $(t, t+h)$ but no birth, no death, and no immigration occur during that interval of time.

f. During $(t, t+h)$ two or more transitions occur with probability $o(h)$, i.e., the occurrence of more than one transition in $(t, t+h)$ is negligible.

All the above contingencies are mutually exclusive and hence for $n \geq 1$, we have

$$
\begin{aligned}
p_{n}(t+h)= & p_{n}(t)[1-n \lambda h+o(h)][1-n \mu h+o(h)][1-v h+o(h)][1-\xi h+o(h)] \\
& +p_{n-1}(t)[\overline{n-1} \lambda h+o(h)][1-\overline{n-1} \mu h+o(h)][1-v h+o(h)][1-\xi h+o(h)] \\
& +p_{n-1}(t)[1-\overline{n-1} \lambda h+o(h)][1-\overline{n-1} \mu h+o(h)][v h+o(h)][1-\xi h+o(h)] \\
& +p_{n+1}(t)[1-\overline{n+1} \lambda h+o(h)][\overline{n+1} \mu h+o(h)][1-v h+o(h)][1-\xi h+o(h)] \\
& +p_{n+1}(t)[1-\overline{n+1} \lambda h+o(h)][1-\overline{n+1} \mu h+o(h)][1-v h+o(h)][\xi h+o(h)] \\
& +o(h), \quad \text { for } \lambda \neq \mu \text { and } v \neq \xi .
\end{aligned}
$$

Hence,

$$
\begin{aligned}
p_{n}^{\prime}(t)= & \lim _{h \rightarrow 0} \frac{p_{n}(t+h)-p_{n}(t)}{h} \\
= & -n \lambda p_{n}(t)-n \mu p_{n}(t)-v p_{n}(t)-\xi p_{n}(t) \\
& +\overline{n-1} \lambda p_{n-1}(t)+v p_{n-1}(t)+\overline{n+1} \mu p_{n+1}(t) \\
& +\xi p_{n+1}(t) \text { as } \lim _{h \rightarrow 0} \frac{o(h)}{h}=0, \text { for } n \geq 1 .
\end{aligned}
$$

For $n=0$, in the similar manner we can find

$$
p_{0}^{\prime}(t)=\xi p_{1}(t)+\mu p_{1}(t)-v p_{0}(t)
$$

We can write (3.2) and (3.3) in a single form by letting $p_{-1}(t)=0, \xi p_{0}(t)=0, \mu p_{0}(t)=0$. Similarly, if the population size reduces to zero at any time point $t$, there will be no emigrations and no death and hence $\xi p_{0}(t)=0, \mu p_{0}(t)=0$. Hence,

$$
\begin{aligned}
p_{n}^{\prime}(t)= & -n \lambda p_{n}(t)-n \mu p_{n}(t)-v p_{n}(t)-\xi p_{n}(t) \\
& +\overline{n-1} \lambda p_{n-1}(t)+\overline{n+1} \mu p_{n+1}(t)+v p_{n-1}(t)+\xi p_{n+1}(t), n \geq 0 .
\end{aligned}
$$

Define the probability-generating function of $p_{n}(t)$ as

$$
\pi(z, t)=\sum_{n=0}^{\infty} p_{n}(t) z^{n}
$$

Hence,

$$
\begin{aligned}
\frac{\partial \pi(z, t)}{\partial t} & =\sum_{n=0}^{\infty} \frac{d p_{n}(t)}{d t} z^{n} \\
& =(\lambda z-\mu)(z-1) \frac{\partial \pi(z, t)}{\partial z}+\frac{(z-1)(\nu z-\xi)}{z} \pi(z, t) .
\end{aligned}
$$

We write this linear partial differential equation given by (3.6), in the form

$$
-\frac{(\lambda z-\mu) z}{v z-\xi} \frac{\partial \log \pi(z, t)}{\partial z}+\frac{z}{(z-1)(v z-\xi)} \frac{\partial \log \pi(z, t)}{\partial t}=1
$$

To solve this partial differential equation, we first form the subsidiary equations given by

$$
\frac{d \log \pi(z, t)}{1}=\frac{d z}{-\frac{z(\lambda z-\mu)}{v z-\xi}}=\frac{d t}{\frac{z}{(z-1)(v z-\xi)}} .
$$


From the first and second parts of (3.8) we find

$$
\frac{\pi(z, t)}{\left(\frac{\lambda z-\mu}{z}\right)^{\frac{\xi}{\mu}}(\lambda z-\mu)^{-\frac{v}{\lambda}}}=\text { a constant. }
$$

From the second and third part of (3.8) we find

$$
\frac{\lambda z-\mu}{z-1} e^{-(\lambda-\mu) t}=\text { a constant }
$$

Hence,

$$
\frac{\pi(z, t)}{\left(\frac{\lambda z-\mu}{z}\right)^{\frac{\xi}{\mu}}(\lambda z-\mu)^{-\frac{v}{\lambda}}}=\psi\left[\frac{\lambda z-\mu}{z-1} e^{-(\lambda-\mu) t}\right],
$$

where $\psi(\cdot)$ is an arbitrary function to be determined by initial conditions.

If the process starts with $n_{0}$ individuals at $t=0$, we have

$$
p_{n_{0}}(0)=1 \text { and } p_{k}(0)=0 \text {, for } k \neq n_{0} \text {. }
$$

And hence $\pi(z, 0)=z^{n_{0}}$. Putting $t=0$ in (3.11), we find

$$
\psi\left[\frac{\lambda z-\mu}{1-z}\right]=\frac{z^{n_{0}}}{\left(\frac{\lambda z-\mu}{z}\right)^{\frac{\xi}{\mu}}(\lambda z-\mu)^{-\frac{v}{\lambda}}}
$$

Letting $u=\frac{\lambda z-\mu}{z-1}$, we find $z=\frac{u-\mu}{u-\lambda}$ and hence from (3.12)

$$
\psi(u)=\frac{\left(\frac{u-\mu}{u-\lambda}\right)^{n_{0}}}{\left[\frac{u(\lambda-\mu)}{u-\mu}\right]^{\frac{\xi}{\mu}}\left[\frac{u(\lambda-\mu)}{u-\lambda}\right]^{-\frac{v}{\lambda}}} .
$$

From (3.11) and (3.13), after simplification, we find

$$
\pi(z, t)=\frac{\left[\left(\frac{\lambda z-\mu}{z-1}-\mu\right) e^{-(\lambda-\mu) t}\right]^{-\frac{\xi}{\mu}}}{\left[\left(\frac{\lambda z-\mu}{z-1}-\lambda\right) e^{-(\lambda-\mu) t}\right]^{-\frac{v}{\lambda}}} \times \frac{\left[\frac{\lambda z-\mu}{z-1} e^{-(\lambda-\mu) t}-\mu\right]^{n_{0}+\frac{\xi}{\mu}}}{\left[\frac{\lambda z-\mu}{z-1} e^{-(\lambda-\mu) t}-\lambda\right]^{n_{0}+\frac{v}{\lambda}}} \text {, for } \lambda \neq \mu \text { and } \nu \neq \xi
$$

Hence, $\pi(z, t)$ given by (3.14) is the probability-generating function of linear growth process with immigration and emigration.

Substituting $z=e^{\theta}$ in the probability-generating function of linear growth process with immigration and emigration given by (3.14) and then find

$$
\begin{aligned}
M(t) & =E[N(t)]=\left.\frac{\partial \pi\left(e^{\theta}, t\right)}{\partial \theta}\right|_{\theta=0}, \text { and } \\
M_{2}(t) & =E[N(t)]^{2}=\left.\frac{\partial^{2} \pi\left(e^{\theta}, t\right)}{\partial \theta^{2}}\right|_{\theta=0}
\end{aligned}
$$

and considering $V[N(t)]=M_{2}(t)-[M(t)]^{2}$, we find the mean and variance of this process given by

$$
\begin{aligned}
E[N(t)]= & \left(\frac{\nu-\xi}{\lambda-\mu}\right)\left[e^{(\lambda-\mu) t}-1\right]+n_{0} e^{(\lambda-\mu) t}, \text { and } \\
V[N(t)]= & \frac{v \mu}{(\lambda-\mu)^{2}}\left[1-e^{(\lambda-\mu) t}\right]+\frac{v \lambda}{(\lambda-\mu)^{2}} e^{(\lambda-\mu) t}\left[e^{(\lambda-\mu) t}-1\right]+\frac{\xi \lambda}{(\lambda-\mu)^{2}}\left[e^{(\lambda-\mu) t}-1\right] \\
& +\frac{\xi \mu}{(\lambda-\mu)^{2}} e^{(\lambda-\mu) t}\left[1-e^{(\lambda-\mu) t}\right]+n_{0}\left(\frac{\lambda+\mu}{\lambda-\mu}\right) e^{(\lambda-\mu) t}\left[e^{(\lambda-\mu) t}-1\right] .
\end{aligned}
$$


Corollary 1. When $\lambda=\mu$ and $\nu=\xi$, from the probability generating function of linear growth process with immigration and emigration given by (3.14), we find

$$
\pi(z, t)=\left[\frac{z(1-\lambda t)+\lambda t}{(1+\lambda t)-\lambda t z}\right]^{n_{0}+\frac{v}{\lambda}} z^{-\frac{v}{\lambda}}
$$

Also from (3.15), we find

$$
E[N(t)]=n_{0} \text { and } V[N(t)]=2 n_{0} \lambda t+2 v t
$$

Corollary 2. If we consider $\lambda_{n}=n \lambda+v$ and $\mu_{n}=n \mu+\xi$, we get also linear growth process with emigration and emigration with same probability-generating function given by (3.14).

\section{DIRECT METHOD TO FIND MEAN AND VARIANCE}

For linear growth process with immigration and emigration, we have

$$
\begin{aligned}
p_{n}^{\prime}(t)= & -n \lambda p_{n}(t)-n \mu p_{n}(t)-v p_{n}(t)-\xi p_{n}(t) \\
& +\overline{n-1} \lambda p_{n-1}(t)+v p_{n-1}(t)+\xi p_{n+1}(t)+\overline{n+1} \mu p_{n+1}(t) .
\end{aligned}
$$

Multiplying both sides of (4.1) by $n$ and summing over all values of $n$, we find

$$
\begin{gathered}
M^{\prime}(t)=(\lambda-\mu) M(t)+(v-\xi) \\
\frac{M^{\prime}(t)}{(\lambda-\mu) M(t)+(v-\xi)}=1 .
\end{gathered}
$$

Hence,

$$
(\lambda-\mu) M(t)+(\nu-\xi)=C_{1} e^{(\lambda-\mu) t},
$$

where $C_{1}$ is a constant to be determined by the appropriate initial conditions.

When the process starts with $n_{0}$ individuals at time $t=0$, we have

$$
p_{n_{0}}(0)=1 \text { and } p_{k}(0)=0 \text {, for } k \neq n_{0}
$$

And hence $M(0)=n_{0}$. Substituting $t=0$ in (4.4), we find

$$
C_{1}=n_{0}(\lambda-\mu)+(\nu-\xi) .
$$

Hence, from (4.4) and (4.5), we find after simplification

$$
M(t)=\left(\frac{\nu-\xi}{\lambda-\mu}\right)\left[e^{(\lambda-\mu) t}-1\right]+n_{0} e^{(\lambda-\mu) t} .
$$

Similarly, multiplying both sides of (4.1) by $n^{2}$ and summing over all values of $n$, we find

$$
\begin{aligned}
M_{2}^{\prime}(t)= & 2(\lambda-\mu) M_{2}(t)+(\lambda+\mu+2 \nu-2 \xi) M(t)+(\nu+\xi) \\
= & 2(\lambda-\mu) M_{2}(t)+(\lambda+\mu+2 \nu-2 \xi)\left[\frac{\nu-\xi}{\lambda-\mu}\left\{e^{(\lambda-\mu) t}-1\right\}+n_{0} e^{(\lambda-\mu) t}\right] \\
& +(\nu+\xi)
\end{aligned}
$$


Hence, the general solution of (4.8) is given by

$$
\begin{aligned}
M_{2}(t)= & C_{2} e^{(\lambda-\mu) t}-\frac{v \lambda}{(\lambda-\mu)^{2}} e^{(\lambda-\mu) t}+\frac{\xi \lambda}{(\lambda-\mu)^{2}} e^{(\lambda-\mu) t}+\frac{v \lambda}{2(\lambda-\mu)^{2}}-\frac{\xi \lambda}{2(\lambda-\mu)^{2}} \\
& -\frac{n_{0} \lambda}{(\lambda-\mu)} e^{(\lambda-\mu) t}-\frac{v \mu}{(\lambda-\mu)^{2}} e^{(\lambda-\mu) t}+\frac{\xi \mu}{(\lambda-\mu)^{2}} e^{(\lambda-\mu) t}+\frac{v \mu}{2(\lambda-\mu)^{2}}-\frac{\xi \mu}{2(\lambda-\mu)^{2}} \\
& -\frac{n_{0} \mu}{(\lambda-\mu)} e^{(\lambda-\mu) t}-\frac{2 v^{2}}{(\lambda-\mu)^{2}} e^{(\lambda-\mu) t}+\frac{2 v \xi}{(\lambda-\mu)^{2}} e^{(\lambda-\mu) t}+\frac{v^{2}}{(\lambda-\mu)^{2}}-\frac{v \xi}{(\lambda-\mu)^{2}} \\
& -\frac{2 n_{0} v}{(\lambda-\mu)} e^{(\lambda-\mu) t}+\frac{2 v \xi}{(\lambda-\mu)^{2}} e^{(\lambda-\mu) t}-\frac{2 \xi^{2}}{(\lambda-\mu)^{2}} e^{(\lambda-\mu) t}-\frac{v \xi}{(\lambda-\mu)^{2}}+\frac{\xi^{2}}{(\lambda-\mu)^{2}} \\
& +\frac{2 n_{0} \xi}{(\lambda-\mu)} e^{(\lambda-\mu) t}-\frac{v}{2(\lambda-\mu)}-\frac{\xi}{2(\lambda-\mu)}
\end{aligned}
$$

If the process starts with $n_{0}$ individuals at time $t=0$, we have $M_{2}(0)=n_{0}^{2}$ and hence substituting $t=0$ in (4.9), we find after simplification

$$
\begin{gathered}
C_{2}=\left[n_{0}^{2}+\frac{\nu \lambda}{(\lambda-\mu)^{2}}-\frac{\xi \lambda}{(\lambda-\mu)^{2}}+\frac{n_{0} \lambda}{(\lambda-\mu)}+\frac{n_{0} \mu}{(\lambda-\mu)}+\frac{\xi}{(\lambda-\mu)}+\frac{2 n_{0} \nu}{(\lambda-\mu)}-\frac{2 n_{0} \xi}{(\lambda-\mu)}\right. \\
\left.+\frac{\nu^{2}}{(\lambda-\mu)^{2}}+\frac{\xi^{2}}{(\lambda-\mu)^{2}}-\frac{2 v \xi}{(\lambda-\mu)^{2}}\right]
\end{gathered}
$$

Substituting the value of $C_{2}$ given by (4.10) in (4.9), we get the value of $M_{2}(t)$.

Since, $V[N(t)]=M_{2}(t)-[M(t)]^{2}$, we find

$$
\begin{aligned}
V[N(t)]= & \frac{v \mu}{(\lambda-\mu)^{2}}\left[1-e^{(\lambda-\mu) t}\right]+\frac{v \lambda}{(\lambda-\mu)^{2}} e^{(\lambda-\mu) t}\left[e^{(\lambda-\mu) t}-1\right]+\frac{\xi \lambda}{(\lambda-\mu)^{2}}\left[e^{(\lambda-\mu) t}-1\right] \\
& +\frac{\xi \mu}{(\lambda-\mu)^{2}} e^{(\lambda-\mu) t}\left[1-e^{(\lambda-\mu) t}\right]+n_{0}\left(\frac{\lambda+\mu}{\lambda-\mu}\right) e^{(\lambda-\mu) t}\left[e^{(\lambda-\mu) t}-1\right] .
\end{aligned}
$$

\section{SOME PARTICULAR CASES}

The suggested linear growth process with immigration and emigration can be reduced to a number of time- -dependent Markov processes for suitable choice of values of $\lambda, \mu, v$, and $\xi$. These are discussed as follows.

\subsection{Linear Birth Process}

Substituting $\mu=0, v=0$, and $\xi=0$, in (3.14), we find the probability generating function of Linear Birth Process given by

$$
\pi(z, t)=\left[\frac{z e^{-\lambda t}}{1-z\left(1-e^{-\lambda t}\right)}\right]^{n_{0}} .
$$

Further letting $\mu=0, v=0$ and $\xi=0$, in (3.15), we find directly the mean and variance of Linear Birth Process given by

$$
E[N(t)]=n_{0} e^{\lambda t} \text { and } V[N(t)]=n_{0} e^{\lambda t}\left(e^{\lambda t}-1\right) .
$$

\subsection{Pure Death Process}

Substituting $\lambda=0, v=0$, and $\xi=0$, in (3.14), we find the probability generating function of Pure Death Process given by

$$
\pi(z, t)=\left[1+(z-1) e^{-\mu t}\right]^{n_{0}}
$$

and from (3.15) the mean and variance of the Pure Death Process are given by

$$
E[N(t)]=n_{0} e^{-\mu t} \text { and } V[N(t)]=n_{0} e^{-\mu t}\left(1-e^{-\mu t}\right) .
$$

\subsection{Immigration Process}

Letting $\lambda \rightarrow 0, \mu \rightarrow 0$, and $\xi=0$, in (3.14), we find the probability generating function of Immigration Process given by

$$
\pi(z, t)=z^{n_{0}} e^{-(1-z) v t}
$$

and from (3.15), we find the mean and variance of the Immigration Process given by

$$
E[N(t)]=n_{0}+v t \text { and } V[N(t)]=v t .
$$




\subsection{Emigration Process}

Substituting $\lambda \rightarrow 0, \mu \rightarrow 0$, and $\nu=0$, in (3.14), we find the probability generating function of the Emigration Process given by

$$
\pi(z, t)=z^{n_{0}} e^{-\left(1-\frac{1}{z}\right) \xi t}
$$

and from (3.15), we find the mean and variance of the Immigration Process given by

$$
E[N(t)]=n_{0}-\xi t \text { and } V[N(t)]=\xi t
$$

\subsection{Linear Growth Process}

Substituting $v=0$ and $\xi=0$, in (3.14), the probability-generating function of Linear Growth Process is given by

$$
\pi(z, t)=\left[\frac{\frac{\lambda z-\mu}{z-1} e^{-(\lambda-\mu) t}-\mu}{\frac{\lambda z-\mu}{z-1} e^{-(\lambda-\mu) t}-\lambda}\right]^{n_{0}}, \text { if } \lambda \neq \mu
$$

and from (3.15) we find the mean and variance of Linear Growth Process given by

$$
E[N(t)]=n_{0} e^{(\lambda-\mu) t} \text { and } V[N(t)]=n_{0} \frac{\lambda+\mu}{\lambda-\mu} e^{(\lambda-\mu) t}\left[e^{(\lambda-\mu) t}-1\right] .
$$

\subsection{Immigration-Emigration Process}

Letting $\lambda \rightarrow 0$ and $\mu \rightarrow 0$, in (3.14), we find the probability generating function of the Immigration-Emigration Process is given by

$$
\pi(z, t)=z^{n_{0}} e^{\frac{(1-z)(\xi-\nu z)}{z} t},
$$

and from (3.15), we find the mean and variance of the Immigration-Emigration Process as

$$
E[N(t)]=n_{0}+(v-\xi) t \text { and } V[N(t)]=(v+\xi) t .
$$

\subsection{Birth-Immigration Process}

Substituting $\mu=0$ and $\xi=0$, in (3.14), we find the probability-generating function of the Birth-Immigration Process given by

$$
\pi(z, t)=\frac{z^{n_{0}} e^{-n_{0} \lambda t} e^{-v t}}{\left[1-z\left(1-e^{-\lambda t}\right)\right]^{n_{0}+\frac{v}{\lambda}}},
$$

and from (3.15), we find the mean and variance of the Birth-Immigration Process as

$$
E[N(t)]=\frac{v}{\lambda}\left(e^{\lambda t}-1\right)+n_{0} e^{\lambda t} \text { and } V[N(t)]=\left(n_{0}+\frac{v}{\lambda}\right) e^{\lambda t}\left(e^{\lambda t}-1\right) .
$$

\subsection{Birth-Emigration Process}

Substituting $\mu=0$ and $v=0$, in (3.14), we find the probability generating function of the Birth-Emigration Process is given by

$$
\pi(z, t)=e^{\frac{z-1}{z}\left(1-e^{\lambda t}\right) \frac{\xi}{\lambda}}\left[\frac{z e^{-\lambda t}}{1-z\left(1-e^{-\lambda t}\right)}\right]^{n_{0}},
$$

and from (3.15), we find the mean and variance of the Birth-Emigration Process as

$$
E[N(t)]=\frac{\xi}{\lambda}\left(1-e^{\lambda t}\right)+n_{0} e^{\lambda t} \text { and } V[N(t)]=\left(n_{0} e^{\lambda t}+\frac{\xi}{\lambda}\right)\left(e^{\lambda t}-1\right) .
$$




\subsection{Death-Immigration Process}

Substituting $\lambda=0$ and $\xi=0$, in (3.14), we find the probability-generating function of the Death-Immigration Process given by

$$
\pi(z, t)=e^{-\frac{v}{\mu}(1-z)\left(1-e^{-\mu t}\right)}\left[1-(1-z) e^{-\mu t}\right]^{n_{0}},
$$

and from (3.15), we get the mean and variance of the Death-Immigration Process as

$$
E[N(t)]=\frac{\nu}{\mu}\left(1-e^{-\mu t}\right)+n_{0} e^{-\mu t} \text { and } V[N(t)]=\left(n_{0} e^{-\mu t}+\frac{\nu}{\mu}\right)\left(1-e^{-\mu t}\right)
$$

\subsection{Death-Emigration Process}

Substituting $\lambda=0$ and $v=0$, in (3.14), we find the probability generating function of the Death-Emigration Process given by

$$
\pi(z, t)=z^{-\frac{\xi}{\mu}}\left[1+(z-1) e^{-\mu t}\right]^{n_{0}+\frac{\xi}{\mu}}
$$

and from (3.15), we find the mean and variance of the Death-Emigration Process as

$$
E[N(t)]=\frac{\xi}{\mu}\left(e^{-\mu t}-1\right)+n_{0} e^{-\mu t} \text { and } V[N(t)]=\left(n_{0}+\frac{\xi}{\mu}\right) e^{-\mu t}\left(1-e^{-\mu t}\right)
$$

\subsection{Linear Growth Process with Immigration}

Substituting $\xi=0$, in (3.14), the probability generating function of Linear Growth Process with Immigration given by

$$
\pi(z, t)=\left[\frac{\left(\frac{\lambda z-\mu}{z-1}-\lambda\right) e^{-(\lambda-\mu) t}}{\frac{\lambda z-\mu}{z-1} e^{-(\lambda-\mu) t}-\lambda}\right]^{\frac{v}{\lambda}} \times\left[\frac{\frac{\lambda z-\mu}{z-1} e^{-(\lambda-\mu) t}-\mu}{\frac{\lambda z-\mu}{z-1} e^{-(\lambda-\mu) t}-\lambda}\right]^{n_{0}}
$$

and from (3.15), we find the mean and variance of Linear Growth Process with Immigration given by

$$
\begin{aligned}
E[N(t)]= & \frac{v}{\lambda-\mu}\left(e^{(\lambda-\mu) t}-1\right)+n_{0} e^{(\lambda-\mu) t} \text { and } \\
= & \frac{v \mu}{(\lambda-\mu)^{2}}\left(1-e^{(\lambda-\mu) t}\right)+\frac{v \lambda}{(\lambda-\mu)^{2}} e^{(\lambda-\mu) t}\left[e^{(\lambda-\mu) t}-1\right] \\
& +n_{0}\left(\frac{\lambda+\mu}{\lambda-\mu}\right) e^{(\lambda-\mu) t}\left[e^{(\lambda-\mu) t}-1\right] .
\end{aligned}
$$

\subsection{Linear Growth Process with Emigration}

Substituting $v=0$, in (3.14), we find the probability generating function of Linear Growth Process with Emigration given by

$$
\pi(z, t)=\left[\frac{\frac{\lambda z-\mu}{z-1} e^{-(\lambda-\mu) t}-\mu}{\left(\frac{\lambda z-\mu}{z-1}-\mu\right) e^{-(\lambda-\mu) t}}\right]^{\frac{\xi}{\mu}} \times\left[\frac{\frac{\lambda z-\mu}{z-1} e^{-(\lambda-\mu) t}-\mu}{\frac{\lambda z-\mu}{z-1} e^{-(\lambda-\mu) t}-\lambda}\right]^{n_{0}}
$$

and from (3.15), we find the mean and variance of Linear Growth Process with Emigration given by

$$
\begin{aligned}
E[N(t)]= & \frac{\xi}{\lambda-\mu}\left(1-e^{(\lambda-\mu) t}\right)+n_{0} e^{(\lambda-\mu) t} \text { and } \\
V[N(t)]= & \frac{\lambda \xi}{(\lambda-\mu)^{2}}\left[e^{(\lambda-\mu) t}-1\right]+\frac{\mu \xi}{(\lambda-\mu)^{2}} e^{(\lambda-\mu) t}\left[1-e^{(\lambda-\mu) t}\right] \\
& +n_{0}\left(\frac{\lambda+\mu}{\lambda-\mu}\right) e^{(\lambda-\mu) t}\left[e^{(\lambda-\mu) t}-1\right] .
\end{aligned}
$$




\subsection{Birth Process with Immigration-Emigration}

Substituting $\mu=0$ in (3.14), we find the probability generating function of Linear Birth Process with Immigration-Emigration given by

$$
\pi(z, t)=\frac{z^{n_{0}} e^{-n_{0} \lambda t} e^{-v t}}{\left[1-z\left(1-e^{-\lambda t}\right)\right]^{n_{0}+\frac{v}{\lambda}}} e^{-\frac{\xi}{\lambda z}\left[1-\left\{1-z\left(1-e^{-\lambda t}\right)\right\} e^{\lambda t}\right] .}
$$

Further, substituting $\mu=0$ in (3.15), we find the mean and variance of Birth Process with Immigration-Emigration given by

$$
\begin{aligned}
E[N(t)] & =n_{0} e^{\lambda t}+\left(\frac{v-\xi}{\lambda}\right)\left(e^{\lambda t}-1\right), \text { and } \\
V[N(t)] & =n_{0} e^{\lambda t}\left(e^{\lambda t}-1\right)+\frac{v}{\lambda} e^{\lambda t}\left(e^{\lambda t}-1\right)+\frac{\xi}{\lambda}\left(e^{\lambda t}-1\right)
\end{aligned}
$$

\subsection{Death Process with Immigration-Emigration}

Substituting $\lambda=0$ in (3.14), we find the probability generating function of Linear Death Process with Immigration-Emigration given by

$$
\pi(z, t)=z^{-\frac{\xi}{\mu}} e^{-\frac{\nu}{\mu}(1-z)\left(1-e^{-\mu t}\right)}\left[1+(z-1) e^{-\mu t}\right]^{n_{0}+\frac{\xi}{\mu}} .
$$

Further, substituting $\lambda=0$ in (3.15), we find the mean and variance of Death Process with Immigration-Emigration given by

$$
\begin{aligned}
& E[N(t)]=n_{0} e^{-\mu t}+\left(\frac{\nu-\xi}{\mu}\right)\left(1-e^{-\mu t}\right), \text { and } \\
& V[N(t)]=n_{0} e^{-\mu t}\left(1-e^{\mu t}\right)+\frac{v}{\mu}\left(1-e^{-\mu t}\right)+\frac{\xi}{\mu} e^{-\mu t}\left(1-e^{-\mu t}\right) .
\end{aligned}
$$

\section{IMPACT OF UNRESTRICTED CONSTANTS ON THE MEAN AND VARIANCE OF LINEAR GROWTH PROCESS WITH IMMIGRATION-EMIGRATION}

For Linear Growth Process with Immigration-Emigration, the mean $M(t)$ is

$$
M(t)=E[N(t)]=\left(\frac{\nu-\xi}{\lambda-\mu}\right)\left[e^{(\lambda-\mu) t}-1\right]+n_{0} e^{(\lambda-\mu) t} .
$$

When $v=\xi$, the immigration and emigration have no control over the population and the mean $M(t)$ of the population distribution tends to a desired value $N$ (say) at a given time $t_{j}$, we have

$$
N=M(t)=E[N(t)]=n_{0} e^{(\lambda-\mu) t_{j}},
$$

so that

$$
N-n_{0} e^{(\lambda-\mu) t_{j}}
$$

is the difference between the means when control and no control respectively are applied.

From (6.1), we have

$$
v-\xi=\frac{(\lambda-\mu)\left[N-n_{0} e^{(\lambda-\mu) t_{j}}\right]}{e^{(\lambda-\mu) t_{j}}-1} .
$$

For all $t_{j}>0$, we have

$$
\frac{\lambda-\mu}{e^{(\lambda-\mu) t_{j}}-1}>0, \forall \lambda \text { and } \mu
$$

If $N>n_{0} e^{(\lambda-\mu) t_{j}}$, we have $\nu-\xi>0$, and hence $v>\xi$, i.e., immigration rate is greater than emigration rate.

If the emigration rate reduces to zero, we have

$$
v=\frac{(\lambda-\mu)\left[N-n_{0} e^{(\lambda-\mu) t_{j}}\right]}{e^{(\lambda-\mu) t_{j}}-1} .
$$


If $N<n_{0} e^{(\lambda-\mu) t_{j}}$, we have $v-\xi<0$, and hence $v<\xi$, i.e., immigration rate is less than emigration rate.

If the immigration rate reduces to zero, we have

$$
\xi=\frac{(\mu-\lambda)\left[N-n_{0} e^{(\lambda-\mu) t_{j}}\right]}{e^{(\lambda-\mu) t_{j}}-1} .
$$

Once we have reached a desired mean $N$ at time $t_{j}$, we may need to maintain the mean at a constant level for all time in future, i.e., $M(t)=N$ for all $t>t_{j}$.

Hence, from (6.4), we have

$$
\nu-\xi=\frac{(\lambda-\mu)\left[N-N e^{(\lambda-\mu)\left(t-t_{j}\right)}\right]}{\left[e^{(\lambda-\mu)\left(t-t_{j}\right)}-1\right]},
$$

for all $t>t_{j}$ i.e.,

$$
v-\xi=(\mu-\lambda) N
$$

If $N>N e^{(\lambda-\mu)\left(t-t_{j}\right)}$, we have $v-\xi>0$ and hence $\lambda<\mu$.

Hence, if the immigration rate reduces to zero, we have

$$
v=(\mu-\lambda) N
$$

If $N<N e^{(\lambda-\mu)\left(t-t_{j}\right)}$, we have $v-\xi<0$ and hence $\lambda>\mu$.

Hence, if the immigration rate reduces to zero, we have

$$
\xi=(\lambda-\mu) N
$$

For linear growth process with immigration and emigration, the variance, $V[N(t)]$ is

$$
\begin{aligned}
V[N(t)]= & \frac{v \mu}{(\lambda-\mu)^{2}}\left[1-e^{(\lambda-\mu) t}\right]+\frac{\nu \lambda}{(\lambda-\mu)^{2}} e^{(\lambda-\mu) t}\left[e^{(\lambda-\mu) t}-1\right]+\frac{\xi \lambda}{(\lambda-\mu)^{2}}\left[e^{(\lambda-\mu) t}-1\right] \\
& +\frac{\xi \mu}{(\lambda-\mu)^{2}} e^{(\lambda-\mu) t}\left[1-e^{(\lambda-\mu) t}\right]+n_{0}\left(\frac{\lambda+\mu}{\lambda-\mu}\right) e^{(\lambda-\mu) t}\left[e^{(\lambda-\mu) t}-1\right] .
\end{aligned}
$$

Since $\frac{e^{(\lambda-\mu) t}-1}{\lambda-\mu}>0$ for all nonnegative $\lambda$ and $\mu$, all the terms in (6.12) are nonnegative except

$$
\frac{v \mu}{(\lambda-\mu)^{2}}\left[1-e^{(\lambda-\mu) t}\right]+\frac{\mu \xi}{(\lambda-\mu)^{2}} e^{(\lambda-\mu) t}\left[1-e^{(\lambda-\mu) t}\right],
$$

which is nonpositive.

So, $V[N(t)]$ is always greater than or equal to zero when $v=0$ and $\xi=0$.

However, when $v$ is nonzero, the greatest value $v$ can have over the interval $\left[t_{0}, t_{j}\right]$ is such as to make $N=0$ is

$$
v_{\max }=n_{0} e^{(\lambda-\mu) t_{j}}\left(\frac{\mu-\lambda}{e^{(\lambda-\mu) t_{j}}-1}\right)
$$

Similarly, when $\xi$ is nonzero, the greatest value $\xi$ can have over the interval $\left[t_{0}, t_{j}\right]$ is such as to make $N=0$ is

$$
\xi_{\text {max }}=n_{0} e^{(\lambda-\mu) t_{j}}\left(\frac{\lambda-\mu}{e^{(\lambda-\mu) t_{j}}-1}\right) .
$$

Consider the terms

$$
\begin{array}{r}
\frac{v \mu}{(\lambda-\mu)^{2}}\left[1-e^{(\lambda-\mu) t_{j}}\right]+\frac{\mu \xi}{(\lambda-\mu)^{2}} e^{(\lambda-\mu) t_{j}}\left[1-e^{(\lambda-\mu) t_{j}}\right] \\
+n_{0}\left(\frac{\lambda+\mu}{\lambda-\mu}\right) e^{(\lambda-\mu) t_{j}}\left[e^{(\lambda-\mu) t_{j}}-1\right]
\end{array}
$$

in $V[N(t)]$. 


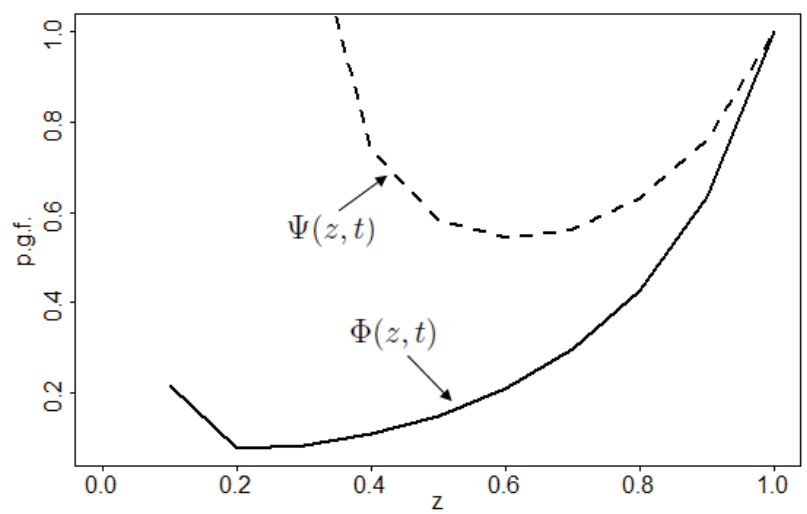

Figure 1 P.G.F. of birth-emigrationprocess.

Substituting the values of $v$ and $\xi$ found by (6.14) and (6.15) respectively in (6.16), we left with

$$
n_{0} \lambda e^{(\lambda-\mu) t_{j}}\left[\frac{e^{(\lambda-\mu) t_{j}}-1}{\lambda-\mu}\right]
$$

which by (6.5) is nonnegative for all nonnegative $\lambda$ and $\mu$.

Hence $V[N(t)]$ is positive for all $t$ and for all possible constant control parameters.

\section{A SIMULATION STUDY}

In the following we performed a simulation study of Birth, Death, Immigration, and Emigration process and some of its particular cases. The behavior of these properties were also discussed except birth, death, and immigration processes because these are most common processes found in various text books.

\subsection{Birth-Emigration Process}

The process depends on the initial population size. Let the process starts with $n_{0}$ individuals. From (5.1) and (5.7), probability generation function of Birth Process and Emigration Process is given by

$$
\Phi(z, t)=z^{n_{0}} e^{-\left(1-\frac{1}{z}\right) \xi t} \times\left[\frac{z e^{-\lambda t}}{1-z\left(1-e^{-\lambda t}\right)}\right]^{n_{0}} .
$$

From (5.15), the p.g.f. of Birth-Emigration Process is

$$
\Psi(z, t)=e^{\frac{z-1}{z}\left(1-e^{\lambda t}\right) \frac{\xi}{\lambda}}\left[\frac{z e^{-\lambda t}}{1-z\left(1-e^{-\lambda t}\right)}\right]^{n_{0}} .
$$

From Figure 1, for the particular values of $\xi, \lambda$ and $n_{0}$, we can get,

$$
\phi(z, t) \leqslant \psi(z, t)
$$

i.e., the p.g.f. of two independent process is less than or equal to the p.g.f. of Birth-Emigration Process. From (5.2) and (5.7), sum of expectation of Birth Process and Emigration Process is given by

$$
M(t)=n_{0}-\xi t+n_{0} e^{\lambda t}
$$

The expectation of Birth-Emigration Process from (5.16) is

$$
M_{1}(t)=\frac{\xi}{\lambda}\left(1-e^{\lambda t}\right)+n_{0} e^{\lambda t}
$$






Figure 2 Mean of birth-emigration process.

From Figure 2, we can get for the particular values of $\xi, \lambda, t$, and $n_{0}$

$$
M(t)=n_{0}-\xi t+n_{0} e^{\lambda t} \geq \frac{\xi}{\lambda}\left(1-e^{\lambda t}\right)+n_{0} e^{\lambda t}=M_{1}(t)
$$

Hence we can conclude that the sum of expectation of Pure Birth Process and Emigration Process is larger than the expectation of BirthEmigration Process, where birth and emigration occurs simultaneously.

\subsection{Chance of Extinction}

The expectation of Birth-Emigration Process is

$$
M_{1}(t)=\frac{\xi}{\lambda}\left(1-e^{\lambda t}\right)+n_{0} e^{\lambda t}
$$

As $t \rightarrow \infty$

$$
M_{1}(t)=\frac{\xi}{\lambda}\left(1-e^{\lambda t}\right)+n_{0} e^{\lambda t}>1
$$

Hence we can conclude that, if $\lambda>\xi$, there is a chance of population explosion. If $\lambda<\xi$, the process will extinct after certain transition and the chance of extinction is nearly unity.

\subsection{Death-Emigration Process}

If the process starts with $n_{0}$ individuals, from (5.3) and (5.7), the probability-generating function of Death Process and Emigration Process is

$$
\Phi_{1}(z, t)=z^{n_{0}} e^{-\left(1-\frac{1}{z}\right) \xi t} \times\left[1+(z-1) e^{-\mu t}\right]^{n_{0}}
$$

From (5.19), the p.g.f. of Death-Emigration Process is

$$
\Psi_{1}(z, t)=z^{-\frac{\xi}{\mu}}\left[1+(z-1) e^{-\mu t}\right]^{n_{0}+\frac{\xi}{\mu}}
$$

These are the p.g.f. within the range $0 \leq z \leq 1$. Let $z_{0}<1$ and $0 \leq t<\infty$. From Figure 3 , we can see that, in the range $0 \leq z \leq z_{0}$

$$
\Phi_{1}(z, t) \geq \Psi_{1}(z, t)
$$

and for the range $z_{0}<z \leq 1$,

$$
\Phi_{1}(z, t) \leqslant \Psi_{1}(z, t) .
$$






Figure 3 P.G.F. of death-emigration process.

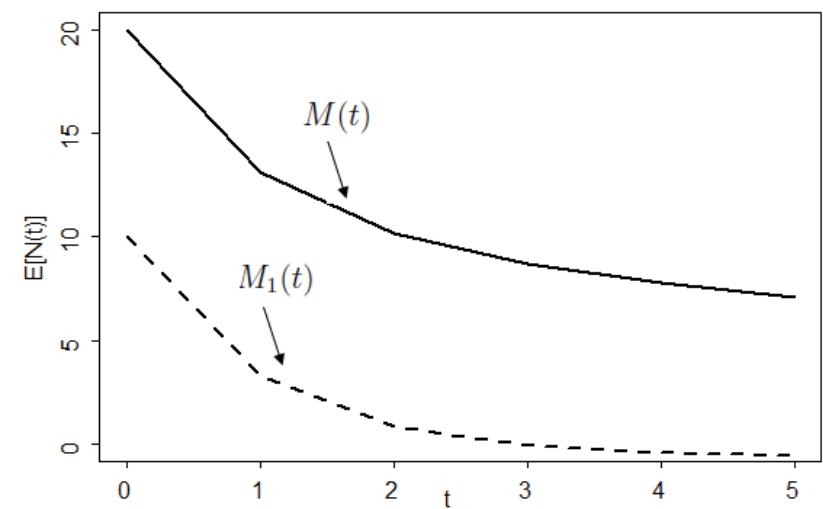

Figure 4 Mean of death-emigration process.

From (5.4) and (5.8), the sum of expectation of Emigration Process and Pure Death Process is

$$
M(t)=n_{0}-\xi t+n_{0} e^{-\mu t}
$$

and from (5.18) the expectation Death-Emigration Process is

$$
M_{1}(t)=\frac{\xi}{\mu}\left(e^{-\mu t}-1\right)+n_{0} e^{-\mu t} .
$$

From Figure 4, we can get,

$$
M(t)=n_{0}-\xi t+n_{0} e^{-\mu t}>\frac{\xi}{\mu}\left(e^{-\mu t}-1\right)+n_{0} e^{-\mu t}=M_{1}(t)
$$

i.e., the sum of expectation of Emigration Process and Pure Death Process is larger than the expectation of Death-Emigration Process.

\subsection{Chance of Extinction}

The expectation of Death-Emigration Process is given by

$$
M_{1}(t)=\frac{\xi}{\mu}\left(e^{-\mu t}-1\right)+n_{0} e^{-\mu t} .
$$

As $t \rightarrow \infty$

$$
M_{1}(t)=\frac{\xi}{\mu}\left(e^{-\mu t}-1\right)+n_{0} e^{-\mu t} \leq 0
$$




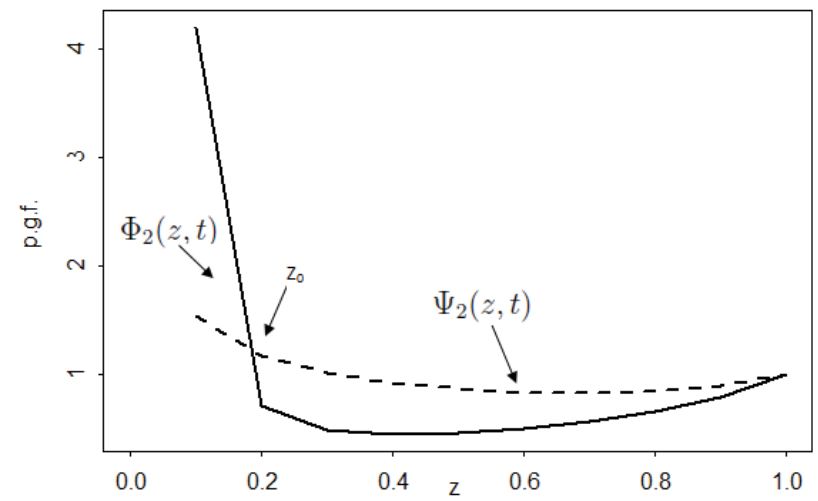

Figure 5 P.G.F. of Birth-Death-Emigration (BDE) process $(\lambda>\mu)$.

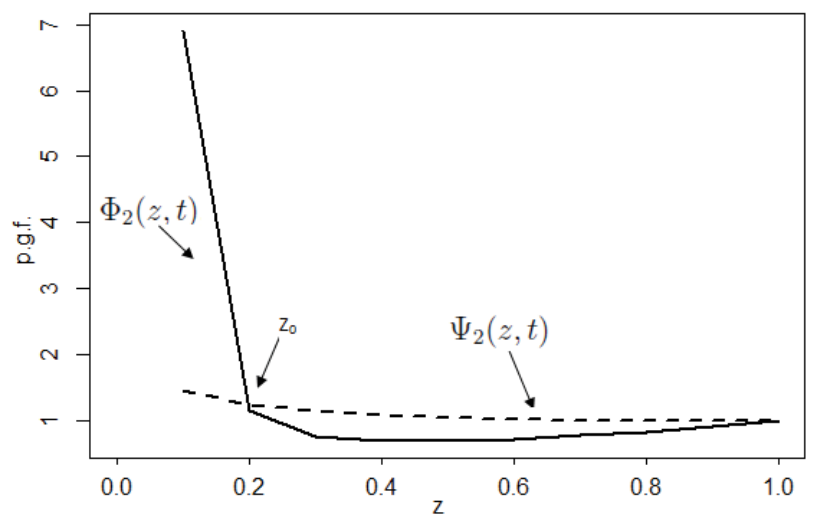

Figure 6 P.G.F. of Birth-Death-Emigration (BDE) process $(\lambda<\mu)$.

i.e., if the process starts with certain individuals, then it will extinct after some transition.

Hence the chance of extinction is nearly unity.

\subsection{Birth-Death-Emigration Process $(\lambda \neq \mu)$}

Let the process starts with $n_{0}$ individuals. From (5.7) and (5.9) the p.g.f. of Emigration Process and Birth-Death Process is

$$
\phi_{2}(z, t)=z^{n_{0}} e^{-\left(1-\frac{1}{z}\right) \xi t} \times\left[\frac{\frac{\lambda z-\mu}{z-1} e^{-(\lambda-\mu) t}-\mu}{\frac{\lambda z-\mu}{z-1} e^{-(\lambda-\mu) t}-\lambda}\right]^{n_{0}}, \text { if } \lambda \neq \mu .
$$

The p.g.f. of Birth-Death-Emigration (BDE) Process from (5.23), is

$$
\psi_{2}(z, t)=\left[\frac{\frac{\lambda z-\mu}{z-1} e^{-(\lambda-\mu) t}-\mu}{\left(\frac{\lambda z-\mu}{z-1}-\mu\right) e^{-(\lambda-\mu) t}}\right]^{\frac{\xi}{\mu}} \times\left[\frac{\frac{\lambda z-\mu}{z-1} e^{-(\lambda-\mu) t}-\mu}{\frac{\lambda z-\mu}{z-1} e^{-(\lambda-\mu) t}-\lambda}\right]^{n_{0}} .
$$

Let $z_{0}<1$ and $0 \leq t<\infty$. From Figures 5 and 6 ), we can see that, in the range $0 \leq z \leq z_{0}$

$$
\Phi_{2}(z, t)>\Psi_{2}(z, t)
$$

and for the range $z_{0}<z \leq 1$,

$$
\Phi_{2}(z, t) \leq \Psi_{2}(z, t)
$$




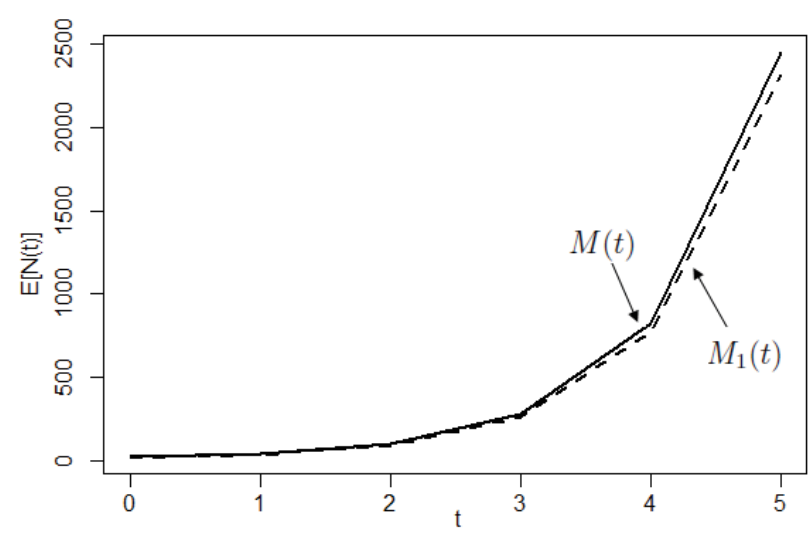

Figure 7 Mean of Birth-Death-Emigration (BDE) process $(\lambda>\mu)$.

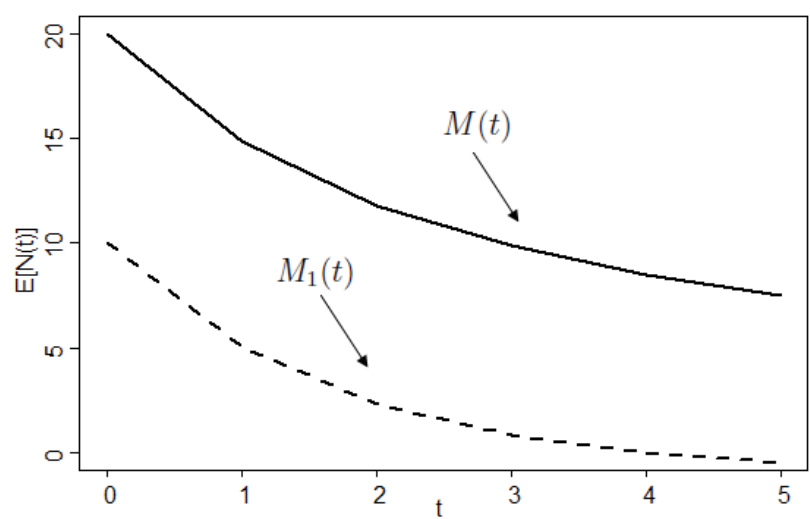

Figure 8 Mean of Birth-Death-Emigration (BDE) process $(\lambda<\mu)$.

From (5.8) and (5.10), the expectation of Emigration Process and Birth-Death Process is given by

$$
M(t)=n_{0}-\xi t+n_{0} e^{(\lambda-\mu) t}
$$

From (5.24), the expectation of BDE Process is given by

$$
M_{1}(t)=\frac{\xi}{\lambda-\mu}\left(1-e^{(\lambda-\mu) t}\right)+n_{0} e^{(\lambda-\mu) t}
$$

From Figures 7 and 8 , we can see if the process starts with $n 0$ individuals, for particular values of $\lambda, \mu$ and $\xi$ when $(\lambda>\mu)$,

$$
M(t)=n_{0}-\xi t+n_{0} e^{(\lambda-\mu) t} \geq \frac{\xi}{\lambda-\mu}\left(1-e^{(\lambda-\mu) t}\right)+n_{0} e^{(\lambda-\mu) t}=M_{1}(t)
$$

and when $(\lambda<\mu)$,

$$
M(t)=n_{0}-\xi t+n_{0} e^{(\lambda-\mu) t}>\frac{\xi}{\lambda-\mu}\left(1-e^{(\lambda-\mu) t}\right)+n_{0} e^{(\lambda-\mu) t}=M_{1}(t)
$$

\subsubsection{Chance of extinction}

The expectation of BDE process is given by

$$
M_{1}(t)=\frac{\xi}{(\lambda-\mu)}\left[1-e^{(\lambda-\mu) t}\right]+n_{0} e^{(\lambda-\mu) t}
$$




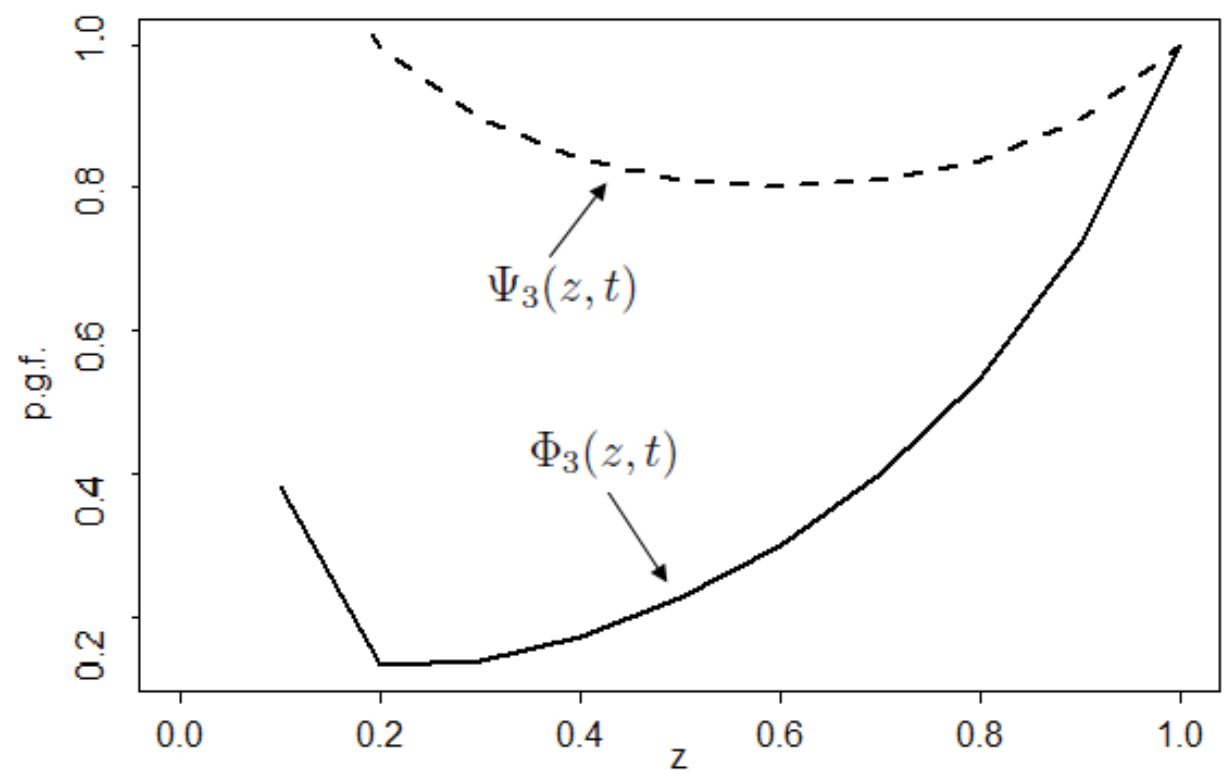

Figure 9 P.G.F. of Birth-Death-Immigration-Emigration (BDIE) process.

As $t \rightarrow \infty$

$$
\begin{aligned}
& M_{1}(t) \simeq n_{0} e^{(\lambda-\mu) t}-\frac{\xi}{(\lambda-\mu)}\left[e^{(\lambda-\mu) t}\right](\lambda>\mu) \\
& M_{1}(t)=\frac{\xi}{(\lambda-\mu)}(\lambda<\mu) .
\end{aligned}
$$

For $(\lambda>\mu)$, As $t \rightarrow \infty M_{1}(t)>1$ but for $(\lambda<\mu)$ As $\mathrm{t} \rightarrow \infty M_{1}(t) \leq 1$

Hence we can conclude that for $(\lambda>\mu)$, there is a chance of population explosion but if the emigration rate will be larger than the birth rate and death rate, then the process will extinct after certain transition. For $(\lambda<\mu)$, the chance of extinction is nearly unity, i.e., the process will extinct after finite number of transition with probability one.

\subsection{Birth-Death-Immigration-Emigration Process $(\lambda \neq \mu)$}

From (5.5), (5.7), and (5.9), the p.g.f. of Immigration Process, Emigration Process, and Birth-Death Process is given by

$$
\phi_{3}(z, t)=z^{n_{0}} e^{-(1-z) v t} \times z^{n_{0}} e^{-\left(1-\frac{1}{z}\right) \xi t} \times\left[\frac{\frac{\lambda z-\mu}{z-1} e^{-(\lambda-\mu) t}-\mu}{\frac{\lambda z-\mu}{z-1} e^{-(\lambda-\mu) t}-\lambda}\right]^{n_{0}}
$$

From (3.14), the p.g.f. Birth-Death-Immigration-Emigration (BDIE) Process is given by

$$
\psi_{3}(z, t)=\frac{\left[\left(\frac{\lambda z-\mu}{z-1}-\mu\right) e^{-(\lambda-\mu) t}\right]^{-\frac{\xi}{\mu}}}{\left[\left(\frac{\lambda z-\mu}{z-1}-\lambda\right) e^{-(\lambda-\mu) t}\right]^{-\frac{\nu}{\lambda}}} \times \frac{\left[\frac{\lambda z-\mu}{z-1} e^{-(\lambda-\mu) t}-\mu\right]^{n_{0}+\frac{\xi}{\mu}}}{\left[\frac{\lambda z-\mu}{z-1} e^{-(\lambda-\mu) t}-\lambda\right]^{n_{0}+\frac{\nu}{\lambda}}}
$$

From Figure 9, We can see

$$
\phi_{3}(z, t) \leqslant \psi_{3}(z, t)
$$

From (5.6), (5.8) and (5.10), the expectation of Immigration Process, Emigration Process, and Birth-Death Process is given by

$$
M(t)=n_{0}+v t+n_{0}-\xi t+n_{0} e^{(\lambda-\mu) t}
$$




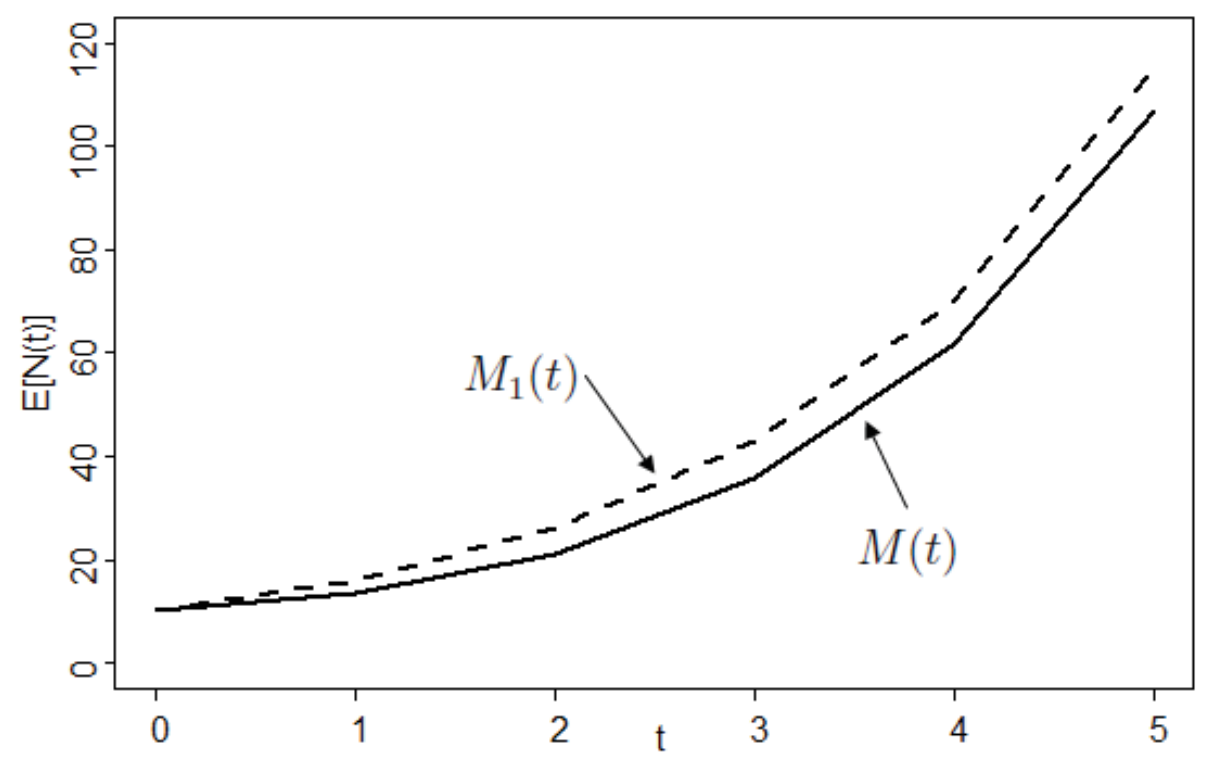

Figure 10 Mean of BDIE process $(\lambda>\mu, \xi>v)$.

and the expectation of BDIE Process $(\lambda \neq \mu)$ is given by

$$
M_{1}(t)=\left(\frac{\nu-\xi}{\lambda-\mu}\right)\left[e^{(\lambda-\mu) t}-1\right]+n_{0} e^{(\lambda-\mu) t}
$$

\section{Case-I $[\lambda>\mu, \xi>v]$}

From Figure 10, we can see, if the process starts with $n_{0}$ individuals for different values of $\xi, \nu, \lambda$, and $\mu$,

$$
M(t)=n_{0}+v t+n_{0}-\xi t+n_{0} e^{(\lambda-\mu) t} \leqslant\left(\frac{\nu-\xi}{\lambda-\mu}\right)\left[e^{(\lambda-\mu) t}-1\right]+n_{0} e^{(\lambda-\mu) t}=M_{1}(t)
$$

i.e., the average growth of BDIE Process is more than the sum of average growth of individual process.

Case-II $[\lambda>\mu, \xi<v]$

From Figure 11, we can see, if the process starts with $n_{0}$ individuals for different values of $\xi, \nu, \lambda$, and $\mu$,

$$
M(t)=n_{0}+v t+n_{0}-\xi t+n_{0} e^{(\lambda-\mu) t} \geq\left(\frac{\nu-\xi}{\lambda-\mu}\right)\left[e^{(\lambda-\mu) t}-1\right]+n_{0} e^{(\lambda-\mu) t}=M_{1}(t)
$$

i.e., the average growth of BDIE Process is less than the sum of average growth of individual process.

Case-III $[\lambda>\mu, \xi=v]$

If the process starts with certain individual then for $(\lambda>\mu, \xi=v)$, from Figure 12, we can see

$$
M(t)=n_{0}+v t+n_{0}-\xi t+n_{0} e^{(\lambda-\mu) t}=\left(\frac{\nu-\xi}{\lambda-\mu}\right)\left[e^{(\lambda-\mu) t}-1\right]+n_{0} e^{(\lambda-\mu) t}=M_{1}(t)
$$

Since $\xi=v$, the expectation of BDIE Process and sum of the expectation of individual process are equal to the expectation of Birth-Death process. As $(\lambda>\mu)$, if the process starts with certain individual, then it shows a positive growth and for large $t$ there is a chance of population explosion.

Case-IV $[\lambda<\mu, \xi<v]$

If the process starts with $n_{0}$ individuals, for different values of $\lambda, \mu, v$ and $\xi$, from Figure 13, we can see

$$
M(t)=n_{0}+v t+n_{0}-\xi t+n_{0} e^{(\lambda-\mu) t} \geq\left(\frac{\nu-\xi}{\lambda-\mu}\right)\left[e^{(\lambda-\mu) t}-1\right]+n_{0} e^{(\lambda-\mu) t}=M_{1}(t)
$$




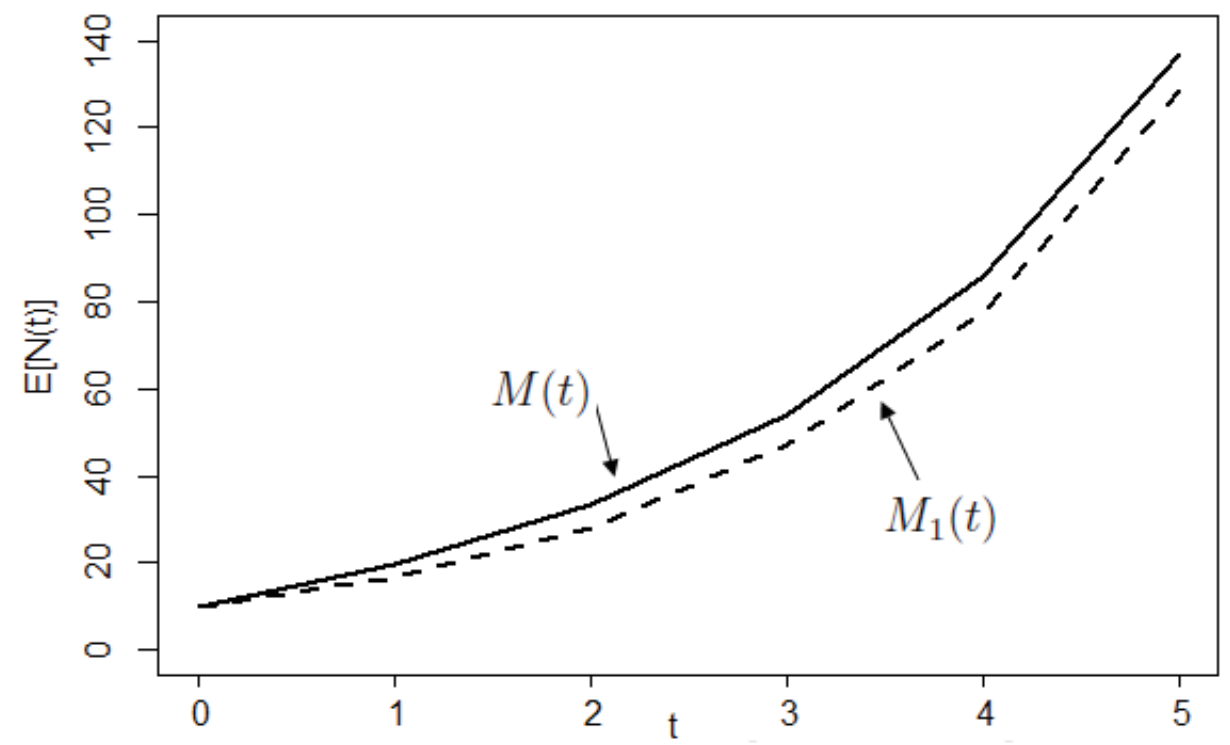

Figure 11 Mean of Birth-Death-Immigration-Emigration (BDIE) process $(\lambda>\mu, \xi<v)$.

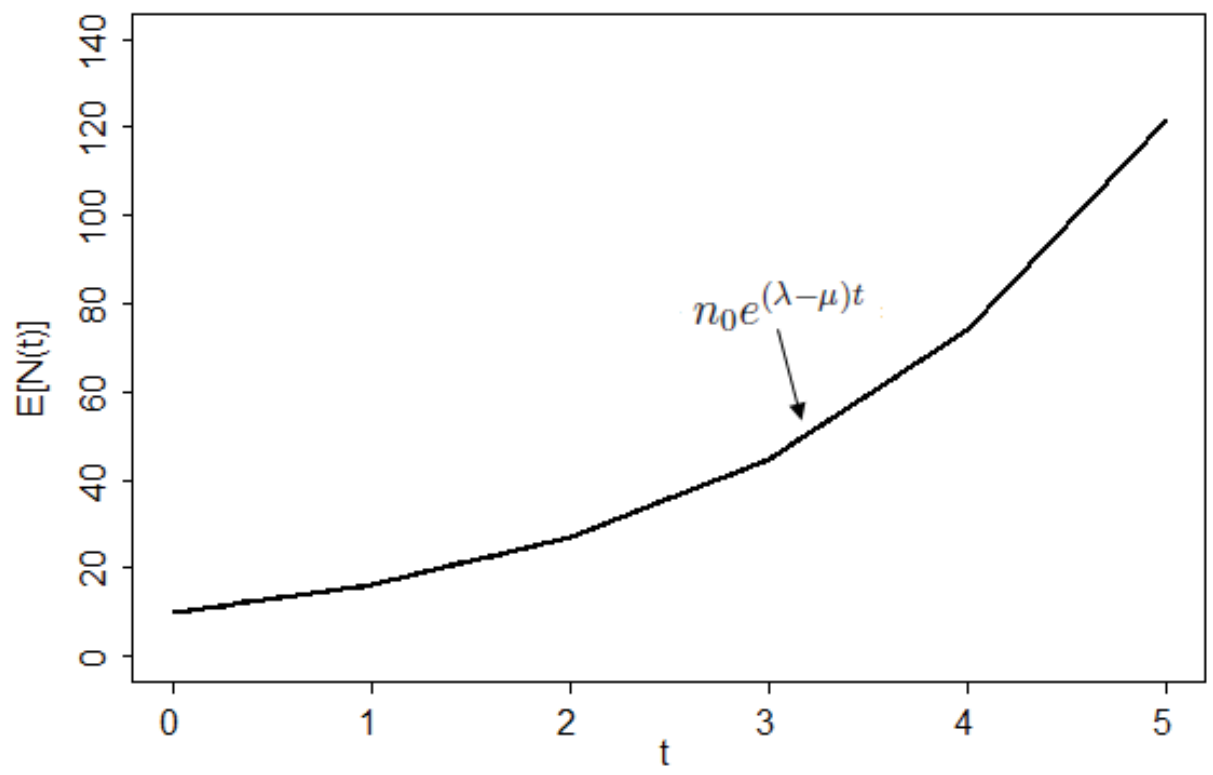

Figure 12 Mean of Birth-Death-Immigration-Emigration (BDIE) process $(\lambda>\mu ; \xi=\nu)$.

Since birth rate is less than the death rate and emigration rate is less than the immigration rate, the sum of expectation of individual process is lager than the expectation of BDIE process the initial population as well as every immigrant leave the system either due to death or emigration. As $(\lambda<\mu)$ here we can expect that the process will extinct after certain transition. Therefore there is a chance of extinction. But in case when we add the expectation of individual process, it grows indefinitely with additional immigrant. So there is chance of population explosion.

Case-V $[\lambda<\mu, \xi>v]$

From Figure 14, if the process starts with $n_{0}$ individuals for different values of $\lambda, \mu, \xi$, and $\nu$ we can see

$$
M(t)=n_{0}+v t+n_{0}-\xi t+n_{0} e^{(\lambda-\mu) t} \leqslant\left(\frac{v-\xi}{\lambda-\mu}\right)\left[e^{(\lambda-\mu) t}-1\right]+n_{0} e^{(\lambda-\mu) t}=M_{1}(t)
$$

i.e., the expectation of the process where birth, death, immigration, and emigration occurs at a time is larger than the sum of expectation of individual process. Since $(\lambda<\mu)$ and $(\xi>v)$, both are shows a negative growth. We can say each process will extinct after certain transition. Hence in both the cases the chance of extinction is nearly unity. 


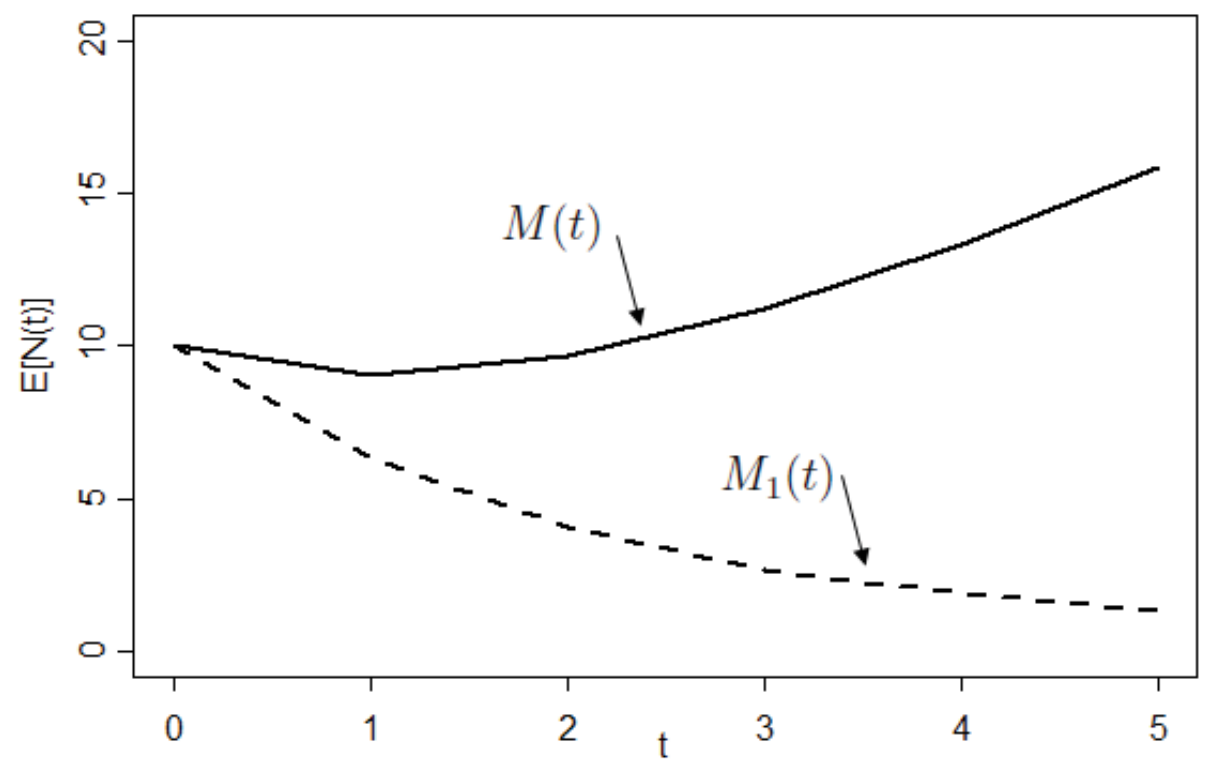

Figure 13 Mean of Birth-Death-Immigration-Emigration (BDIE) process $(\lambda<\mu ; \xi<v)$.

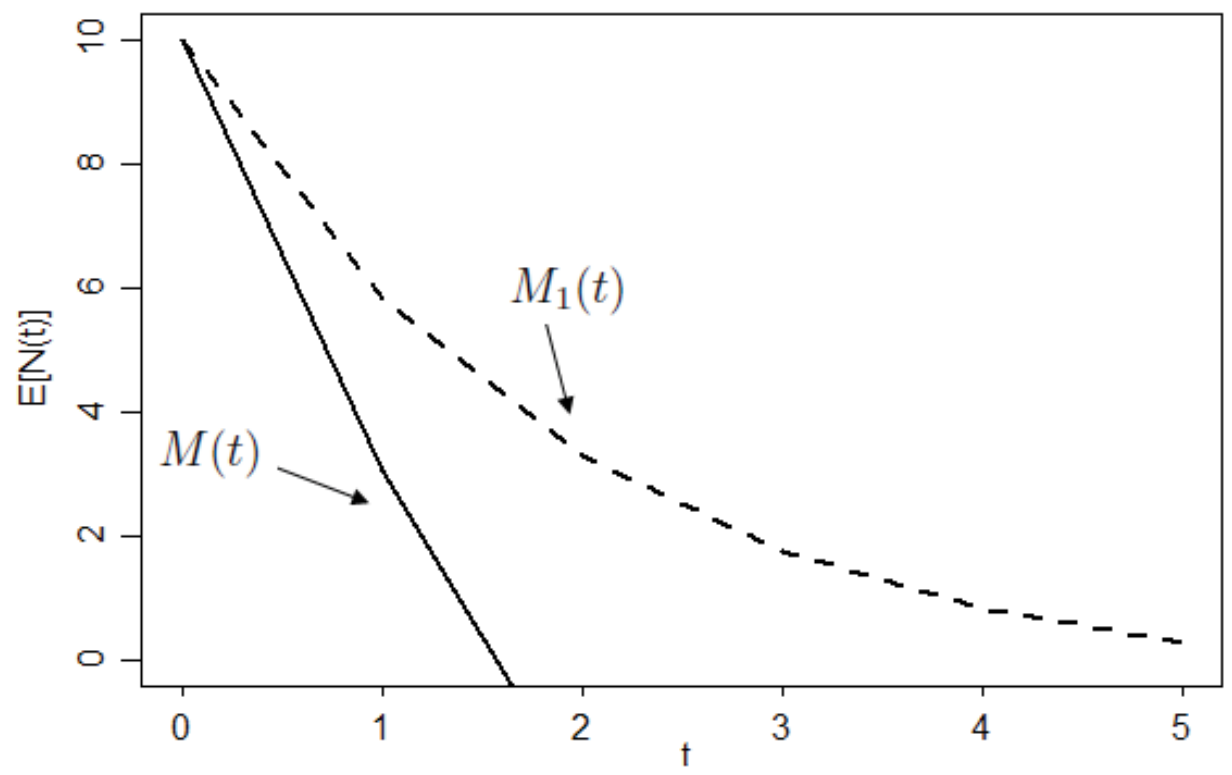

Figure 14 Mean of Birth-Death-Immigration-Emigration (BDIE) process $(\lambda<\mu$; $\xi>v)$.

Case-VI $[\lambda<\mu, \xi=v]$

For $(\lambda<\mu)$ and $(\xi=\nu)$ Figure 15, indicates that the process will become the expectation of Birth-Death Process and

$$
M(t)=n_{0}+v t+n_{0}-\xi t+n_{0} e^{(\lambda-\mu) t}=\left(\frac{\nu-\xi}{\lambda-\mu}\right)\left[e^{(\lambda-\mu) t}-1\right]+n_{0} e^{(\lambda-\mu) t}=M_{1}(t)
$$

Since in both the case it becomes the expectation of Birth-Death Process $(\lambda<\mu)$, it shows a negative growth and process will extinct after certain transition. 


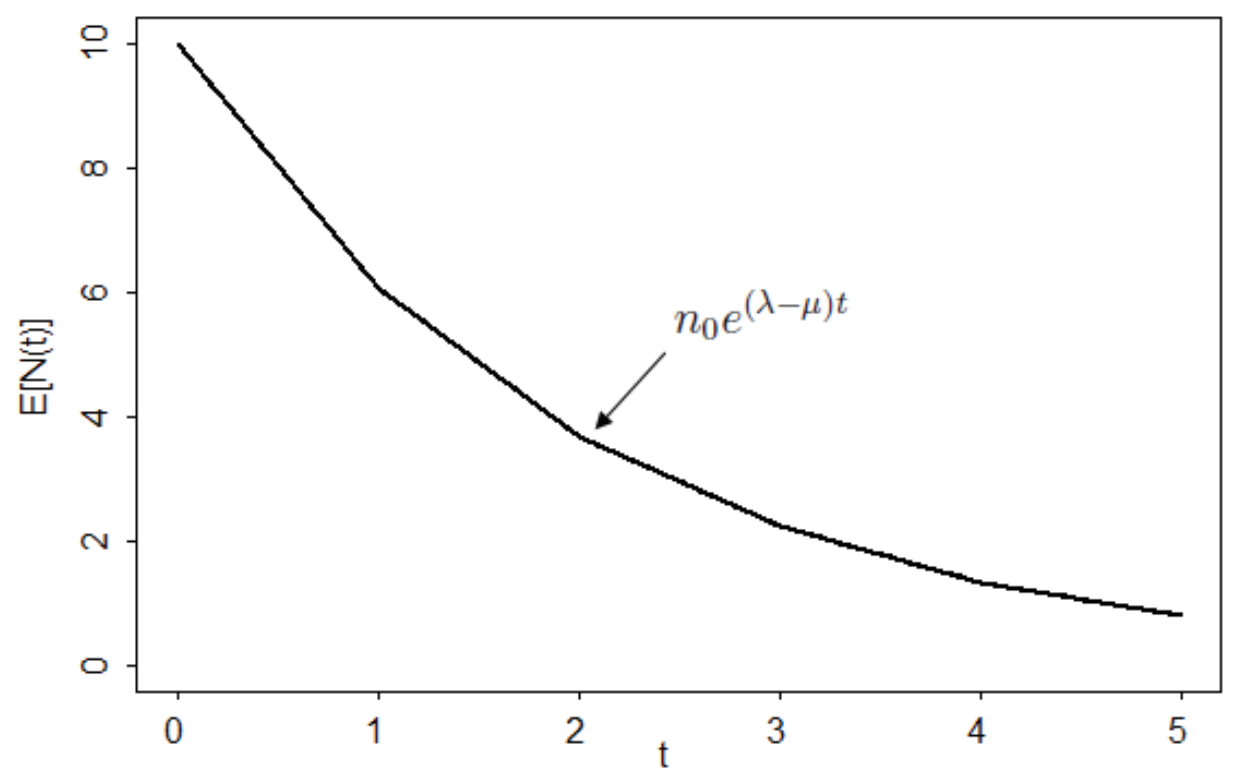

Figure 15 Mean of Birth-Death-Immigration-Emigration (BDIE) process $(\lambda<\mu$; $\xi=v)$.

\subsection{Chance of Extinction}

The expectation of BDIE process is given by

$$
M_{1}(t)=\frac{v}{(\lambda-\mu)}\left[e^{(\lambda-\mu) t}-1\right]-\frac{\xi}{(\lambda-\mu)}\left[e^{(\lambda-\mu) t}-1\right]+n_{0} e^{(\lambda-\mu) t}
$$

As $t \rightarrow \infty$

$$
M_{1}(t)= \begin{cases}\frac{v}{(\lambda-\mu)}\left[e^{(\lambda-\mu) t}-1\right]-\frac{\xi}{(\lambda-\mu)}\left[e^{(\lambda-\mu) t}-1\right]+n_{0} e^{(\lambda-\mu) t}, & \lambda>\mu \\ \frac{v}{(\lambda-\mu)}+\frac{\xi}{(\lambda-\mu)}, & \text { if } \lambda<\mu\end{cases}
$$

Hence we can conclude that as $t \rightarrow \infty$, the chance of extinction and chance of Population explosion is depends on $(\lambda>\mu)$ and $(\lambda<\mu)$. If $(\lambda>\mu)$, there is a chance of population explosion and if $(\lambda<\mu)$, the chance of extinction is nearly unity.

\section{CONCLUSION}

The process discussed in this paper is the linear growth process with immigration and emigration, which is a generalization to several population processes. The probability generating function of this process is also derived. Mean and variance are obtained by direct method and also from the probability generating function. Several other processes are also derived as particular cases with their probability generating functions. Also, the impact of unrestricted constants on the mean and variance of this process are discussed. The behavior of different processes are also analyzed using a simulation study. The chance of extinction of these processes were also studied.

\section{CONFLICTS OF INTEREST}

The authors declare that there is no conflict of interest.

\section{AUTHORS' CONTRIBUTIONS}

All the authors are contributed for the derivation of expressions in the research article and Upasana has contributed towards the simulation study in the article.

\section{Funding Statement}

The authors have not received ant fund for the preparation of this manuscript from any agency or organization. 


\section{ACKNOWLEDGMENTS}

The authors are very much thankful to the reviewers for their valuable inputs to bring this research paper to this form.

\section{REFERENCES}

1. W.M. Getz, Math. Biosci. 23 (1975), 87-111.

2. Y. Zheng, X. Chao, J. Xiaomei, Probab. Eng. Informat. Sci. 18 (2004), 141-159. 